\title{
Fitting patient-generated health data for women into their personal health plans: VA has an app for that!
}

\author{
Kathleen L Frisbee*
}

U.S. Department of Veterans Affairs, USA

Women Veterans are the fastest-growing Veteran population: As of 2015, approximately 2 million Veterans in the United States and Puerto Rico were women. As the number of women Veterans increases, the U.S. Department of Veterans Affairs (VA) continues to prepare for an increasing demand for women Veterans' health care needs.

With the rapid pace of modern life, it's no surprise that Veterans and non-Veterans alike have turned to consumer health products to help monitor their own health. Devices such as Apple Watches, Fitbits, and more provide a wealth of patient generated data. But there often exists a disconnect between the personally selected activities that patients engage in to improve their health and the clinically prescribed activities that happen as a result of a visit to a provider.

According to research from Pew, one in five American adults use a smart watch or a fitness tracker, and women are more likely to use a fitness device than men. VA's Office of Connected Care (OCC), which focuses on integrating mobile health technology with Veteran care, wants to make sure that women Veterans make good use of the information gathered from these devices by working with their care teams toward better health. We saw the proliferation of these popular new devices as an opportunity to provide VA health teams with information about their patient's health between face-to-face visits.

For example, we know from the Centers for Disease Control and Prevention that cardiovascular disease, which includes heart disease, hypertension, and stroke, is the No. 1 killer of women in the United States. It's often referred to as a "silent killer" of women as some may not experience the typically associated symptoms. Through VA OCC technologies, we're blending VA telehealth technology and patientgenerated data to promote heart health and potentially even detect health risks sooner. An example of this is our Apple Watch pilot program. A group of Veterans at James A. Haley Veterans' Hospital in Tampa, Florida, have each received an Apple Watch that can conduct an electrocardiogram (EKG) - a test that monitors the heart's electrical signal - and save the results in a format that makes them shareable with the Veteran's VA care team.

This electronic transfer of information often saves Veterans from having to drive to in-person appointments at the VA Medical Center. Once a Veteran is able to transfer the EKG results to the Health app on their iOS device, they can then share it with their provider through one of two VA innovations: during a VA Video Connect appointment, or through Secure Messaging on My HealtheVet, VA's online patient portal.

In addition to Apple Watches, VA is gathering data from other types of Connected Devices including FitBits and Glucometers. OCC has developed a mobile app called "Sync-My-Health-Data" (SMHD), which allows a Veteran to pair their device with the SMHD app so that the data can flow into VA's Patient Generated Database for analysis. OCC is conducting an analysis of this data, coupled with data from VA's Electronic Health Record and Socioeconomic data for two clinical cohorts - Diabetes and Sleep/Stress. The insights generated from this analysis will be presented back to VA providers and patients.

While its helpful to incorporate all these tools into the care plans of our Veterans, evidence shows that many patients prefer simple text messaging. Our text messaging application, Annie is a great example of VA technology enhancing patient care by integrating patient generated health data. Named after Lt. Annie G. Fox, the first woman to receive the Purple Heart for combat, Annie uses automated text messaging to promote self-care for Veterans enrolled in VA health care.

Here's how it works: when a patient is enrolled in a clinical protocol, such as medication management, the Annie app can be programmed to send personalized, automated text messages to the patient to remind them to take their medications.

By using Annie, patients who are enrolled in hypertension management can send their blood pressure readings back to their clinical care team via a text message. Annie will alert Veterans if the readings are outside their desired ranges. Users can also receive exercise reminders to encourage a healthy lifestyle and help lower the risk of cardiovascular disease.

VA has over 6,000 active patients using Annie, 22\% of which are female Veterans, to manage a variety of clinical conditions, and the number of users is steadily growing. And excitement among VA providers about Annie is growing too.

"VA providers should be wholeheartedly enthusiastic about using Annie. It allows us to reach our patients in ways we couldn't previously and ultimately improve health outcomes," said Dr. Lynn Kataria, chief of neurology education at the Washington, D.C. VA Medical Center in a VA testimonial.

Annie is an excellent tool to engage patients in their own care, and acts as a reminder for self-care. Using Annie allows providers the ability to review patient data at any time prior to the patient's visit, enabling them to adjust the plan of care if necessary.

VA OCC is continuously developing new technology solutions to enhance our disease management and prevent capabilities for the Veterans we serve. In the coming months we're excited to begin rolling

${ }^{\star}$ Correspondence to: Kathleen L. Frisbee, U.S. Department of Veterans Affairs, USA, Tel: 844-698-2311; E-mail: Kathleen.Frisbee@va.gov

Received: January 13, 2020; Accepted: January 27, 2020; Published: January 30, 2020 
out our new FitHeart mobile application. FitHeart will help Veterans improve their heart health by enabling them to set weekly fitness goals and exercise reminder notifications, record fitness activities, and track heart health measures including blood pressure, weight, and blood sugar levels.
Virtual health is just one way we're improving health care services for all who have served. To learn more about how the VA Office of Connected Care is using technology to improve health care for Veterans, visit https://connectedcare.va.gov/.

Copyright: $\bigcirc 2020$ Frisbee KL. This is an open-access article distributed under the terms of the Creative Commons Attribution License, which permits unrestricted use, distribution, and reproduction in any medium, provided the original author and source are credited. 